Journal of Porous Materials, Volume 21, Issue 4, pp 485-493 (2014)

DOI: 10.1007/s10934-014-9795-6 


\title{
TRANSFORMATION OF ETHYLBENZENE-M-XYLENE MIXTURE ON ZEOLITES WITH DIFFERENT STRUCTURES
}

\author{
R.M. Mihályi ${ }^{\text {a, }}$, Sz. Klébert ${ }^{\text {a }}$, M. Kollár ${ }^{\text {a }}$, V. Mavrodinova ${ }^{\text {b }}$ \\ ${ }^{a}$ Institute of Materials and Environmental Chemistry, Research Centre for Natural Sciences, \\ Hungarian Academy of Sciences, Pusztaszeri ut 59-67, Budapest 1025, Hungary \\ b Institute of Organic Chemistry with Centre of Phytochemistry, Bulgarian Academy of \\ Sciences, Acad. G. Bonchev str., bl. 9, 1113 Sofia, Bulgaria
}

\begin{abstract}
Hierarchical zeolite ZSM-5 synthesized by applying amphiphilic organosilane as mesopore template, nanosized zeolite Beta and zeolite MCM-22 have been studied, for the first time in the conversion of mixed ethylbenzene-m-xylene feed. The effects of the channel structure, nanosizing and presence of mesopores in these zeolite materials with close $\mathrm{Si} / \mathrm{Al}$ molar ratio on the catalytic activity and selectivity have been discussed. It was found that the diverse zeolites have different advantages and disadvantages in dependence on their structure and morphology. MCM-22 zeolite provides promising ethylbenzene conversion at low xylene loss with high production of the p-isomer among xylene.
\end{abstract}

Keywords: Hierarchical ZSM-5; nanosized Beta; zeolite MCM-22; conversion of ethylbenzene-m-xylene mixed feed

\section{Introduction}

The main feed stocks for manufacturing of the valuable $\mathrm{p}$-xylene product are mixtures of $\mathrm{C}_{8^{-}}$ aromatics containing o-xylene, m-xylene and 20 to $40 \%$ ethylbenzene (EB), produced in catalytic reforming of naphtha and naphtha pyrolysis. The transformation of this mixed feed on acidic catalysts should be mainly directed to isomerization of the xylenes ingredients up to the equilibrium value and to $\mathrm{EB}$ conversion to valuable products with a minimum loss of xylenes. Two types of commercial zeolites are used for the isomerization of $\mathrm{C}_{8}$ aromatic cut. In the process applying mordenite-based, large-pore zeolite as bifunctional catalyst, EB is mainly converted into xylenes, whereas EB dealkylation and disproportionation are the main reaction routes over medium-pore pentasil-based catalysts (ZSM-5) [1, 2].

\footnotetext{
* Corresponding author. Tel.: 36-1-438-1100; fax: 36-1-325-7554.

E-mail address: mihalyi.magdolna@ttk.mta.hu
} 
Nowadays strategies to enhance the effectiveness of zeolite catalysts essentially comprise preparation of materials with wide pores and/or with short diffusion length. Available approaches are reviewed according to the principle, versatility, effectiveness, and degree of reality for practical implementation of the resulting materials as well as to assessment of their catalytic function [3-5]. In his thorough review Holm et al. [5] claimed that although there is still a long way to go for commercialization and adapting the newly born hierarchical zeolite materials in industrial processes, the application of the nanozeolite and their hierarchical assemblies have shown their potentials with some of the emerging, exciting examples in catalysis and biology.

Modification of the zeolite topology by different in-situ and ex-situ procedures for mesopore formation plays an important role on the catalytic performance in the reactions of alkylaromatics transformations. The catalytic activity in reactions like aromatics alkylation [69] and toluene disproportionation over hierarchical ZSM-5 prepared by templating method [9] or xylenes isomerization [10] on the same desilicated zeolite, have been found to be enhanced compared to their microporous analogues.

The catalytic performance of nanosized zeolites providing external mesoporosity is comparatively less studied in reactions of alkylaromatics transformations. Acid site distribution over nanoscale ZSM-5 materials has been thoroughly studied by K. Wang et al. [11-13] together with their catalytic activity upon toluene disproportionation and 1,2,4- and 1,3,5-trimethylbenzenes cracking. According to the authors, the increased accessibility and the presence of higher percent of external acid sites located on the nanoparticles surface are the reason for the higher reactivity of the bulkier reactants participating in the catalytic act.

Dealkylation and transalkylation of ethylbenzene and its mixtures with xylenes was found to be influenced by variation in the zeolite topology by direct synthesis of zeolites with channel systems containing $10 \mathrm{MR}, 12 \mathrm{MR}$ and $10+12 \mathrm{MR}$ [14] or pore size regulation by liquid phase TEOS deposition [15]. According to our knowledge, however, the influence of the simultaneous presence of micro and mesopores in the so-called hierarchical zeolite materials has not been studied for mixed ethylbenzene-m-xylene reactant feed. In the patent literature only a separate ethylbenzene and m-xylene conversions over mesoporous ZSM-5 synthesized with carbon black matrix is described [16].

The synthesis of zeolite MCM-22 patented by Mobil in 1990 [17] was followed by its fast industrial application for production of EB through liquid-phase alkylation of benzene with ethylene at low temperature. The reverse reaction over the same catalyst is only scarcely studied [18] while that of the conversion of $\mathrm{C}_{8}$ mixed isomerization feed has not been 
investigated at all, although MCM-22 has channel dimensions similar to those of the two commercial zeolite catalysts for $\mathrm{C}_{8}$ feed isomerization. Several types of zeolites, bifunctional or not, with different structures and acidity characteristics have been examined in the latter process [15,19-22], but not with zeolite MCM-22.

The structure of MCM-22 is between that of the medium-pore (10-MR) and of the largepore (12-MR) zeolites as it is built from independent 10-MR channels and supercages with 10-MR entrance, as well as 12-MR cups located on the surface of the crystallites. Thus, such a structure seems promising since it could combine the selective elimination of EB by dealkylation with the preferential xylene isomerization over disproportionation.

In the present work the transformation of ethylbenzene-m-xylene mixed feed over zeolites with three different pore structures, modified morphology and texture has been compared. Medium-pore ZSM-5 with hierarchical structure, nanosized large-pore BEA and MCM-22 with both medium and large pores has been investigated. The effects of the formation of mesopores beside the micropores in the same crystallites in case of ZSM-5, as well as that of nanosizing for BEA on the catalytic behavior are also examined.

\section{Experimental}

\subsection{Catalysts preparation}

For the synthesis of the hierarchical ZSM-5, designated as ZSM-5 (H), [3-(trimethoxysilyl) propyl] hexaoctyldimethylammonium chloride (TPHOC) (Aldrich) mesopore template was added to a conventional alkaline mixture containing TPABr (Fluka), $\mathrm{NaOH}$ (Reanal), tetraethylorthosilicate (98\%, Aldrich), sodium aluminate (Wako) according to the procedure reported in Ref. [23]. The molar composition of the synthesis gel was $1 \mathrm{Al}_{2} \mathrm{O}_{3}: 40 \mathrm{SiO}_{2}: 1.6$ TPHOC : $5.0 \mathrm{Na}_{2} \mathrm{O}: 10 \mathrm{TPABr}: 7200 \mathrm{H}_{2} \mathrm{O}$. A conventional ZSM-5 reference sample, designated as ZSM-5 (R) was synthesized using the same synthesis composition as used for the ZSM-5 (H) sample, but without the silane surfactant (Table 1). In both cases the synthesis gel was hydrothermally treated at $443 \mathrm{~K}$ for 72 hours.

The BEA sample was prepared according to Wadlinger et al. [24] using water glass and silica gel, as well as $\mathrm{Al}_{2}\left(\mathrm{SO}_{4}\right)_{3} \cdot 18 \mathrm{H}_{2} \mathrm{O}$ as silicon and aluminum source, respectively. Tetraethyl ammonium hydroxide (TEA) (Aldrich) was applied as structure directing agent. The crystallization of the synthesis gel with the molar composition of $1 \mathrm{Al}_{2} \mathrm{O}_{3}: 35.5 \mathrm{SiO}_{2}$ : $1.8 \mathrm{Na}_{2} \mathrm{O}: 2.9 \mathrm{Na}_{2} \mathrm{SO}_{4}: 7.2(\text { TEA) })_{2} \mathrm{O}: 450 \mathrm{H}_{2} \mathrm{O}$ was carried out at $409 \mathrm{~K}$ for 190 hours in static condition. Two beta samples with different crystallite sizes were recovered from the 
crystallization product. The BEA material having larger crystals obtained by low-speed centrifugation (8 $000 \mathrm{rpm}, 90 \mathrm{~min}$.) was designated as $B E A(R)$. The remaining supernatant liquid was a stable colloidal suspension, and the other BEA sample was separated from this suspension by high-speed centrifugation (20 $000 \mathrm{rpm}, 90 \mathrm{~min}$.) and designated as BEA(nano) (Table 1).

The as-synthesized zeolite samples were filtered, washed with distilled water, and dried at $393 \mathrm{~K}$. The templates were removed by calcinations in air at $813 \mathrm{~K}$ for 6 hours. After template removal the sodium-form was obtained. To get the $\mathrm{NH}_{4}$-form, the materials were treated three times with $1 \mathrm{M} \mathrm{NH}_{4} \mathrm{Cl}$ solution at room temperature. The $\mathrm{H}$-forms were obtained by in situ decomposition of the $\mathrm{NH}_{4}$-forms prior to each experiment.

Table 1. The chemical composition, IEC and textural properties of the zeolite samples

\begin{tabular}{cccccccc}
\hline Sample & $\begin{array}{c}\mathrm{Si} / \mathrm{Al} \\
\text { bulk }\end{array}$ & $\begin{array}{c}\mathrm{Al} \\
\mathrm{mmol} / \mathrm{g}\end{array}$ & $\begin{array}{c}\mathrm{Na} \\
\mathrm{mmol} / \mathrm{g}\end{array}$ & $\mathrm{IEC}^{\mathrm{b}}$ & $\begin{array}{c}\text { Specific } \\
{\text { surface } \mathrm{area}^{\mathrm{c}}}_{\mathrm{\textrm {m } ^ { 2 } / \mathrm { g }}}\end{array}$ & $\begin{array}{c}\text { Micropore } \\
\text { volume }^{\mathrm{d}} \\
\mathrm{cm}^{3} / \mathrm{g}\end{array}$ & $\begin{array}{c}\text { Mesopore } \\
\text { volume }^{\mathrm{d}} \\
\mathrm{cm}^{3} / \mathrm{g}\end{array}$ \\
\hline ZSM-5(R) & 16.8 & 0.99 & 0.12 & 0.72 & 394 & 0.14 & - \\
ZSM-5(H) & 16.8 & 1.03 & 0.16 & 0.56 & 870 & 0.09 & 0.22 \\
BEA(R) & 12.6 & 1.26 & 0.13 & 1.16 & 630 & 0.24 & - \\
BEA(nano) & 12.6 & 1.18 & 0.11 & 1.02 & 670 & 0.22 & 0.08 \\
MCM-22 & 16.5 & 1.08 & 0.03 & 0.96 & 578 & 0.18 & - \\
\hline
\end{tabular}

${ }^{a}$ All data were related to $1 \mathrm{~g}$ of sample calcined at $1273 \mathrm{~K}$.

${ }^{b}$ The ion exchange capacity (IEC) of the sample, obtained as the amount of ammonia, evolved from the $\mathrm{NH}_{4}{ }^{+}$-form sample in the 453-923 $\mathrm{K}$ temperature range during the TPAE run.

${ }^{c}$ Calculated from the $B$ point of the adsorption isotherm.

${ }^{d}$ Calculated from the $\alpha_{s}$ plots.

The [Al]MCM-22 precursor was prepared by hydrothermal synthesis applying rotating industrial autoclave $\left(\sim 1 \mathrm{~m}^{3}\right)$ according to the procedure described in Ref. [25]. The crystallization temperature and time were $418 \mathrm{~K}$ and 10 days respectively. After washing with distilled water and drying at $343 \mathrm{~K}$, the the resulting solid was calcined in air (programmed heating, $1.5 \mathrm{~K} / \mathrm{min}$ up to $823 \mathrm{~K}$ for $3 \mathrm{~h}$ ) in order to remove the template. Twofold ion exchange with $1 \mathrm{M} \mathrm{NH}_{4} \mathrm{NO}_{3}$ solution was applied for the preparation of its ammonium form. The H-form of the sample with $\mathrm{Si} / \mathrm{Al}$ molar ratio of 16 was obtained by in situ decomposition of the $\mathrm{NH}_{4}{ }^{+}$-form in $\mathrm{N}_{2}$ at $823 \mathrm{~K}$ and was indicated as MCM-22, (Table 1). 


\subsection{Characterization}

The aluminum and sodium contents of the samples were determined by atomic absorption spectroscopy (AAS) after dissolving the solids in hydrofluoric acid. The sum of $\mathrm{Na}_{2} \mathrm{O}$ and $\mathrm{Al}_{2} \mathrm{O}_{3}$ contents was subtracted from the total mass of the analyzed samples to get the silica content.

The XRD patterns were recorded using Phillips PW 1810 type diffractometer, equipped with graphite monochromator. The $\mathrm{Cu} \mathrm{K}_{\alpha}$ radiation was applied.

The crystal size and the morphology were examined using SEM (Hitachi).

The isotherms of $\mathrm{N}_{2}$ adsorption were determined at $77 \mathrm{~K}$ using a Quantachrome Autosorb-1 Automated Gas Sorption Instrument. Before measurements the samples were degassed by evacuation at $623 \mathrm{~K}$ for $12 \mathrm{~h}$. The specific surface area was calculated by the $\mathrm{B}$ point method. The micro and mesopore volumes were estimated from the $\alpha_{\mathrm{s}}$ plots (Table 1).

The $\mathrm{NH}_{4}{ }^{+}$-ion exchange capacity (IEC) of the samples were characterized by TPAE (Temperature-Programmed Ammonia Evolution) measurements described elsewhere [26] and presented in Table 1. About $300 \mathrm{mg}$ of samples were heated from 423 to $823 \mathrm{~K}$ at a rate of $10 \mathrm{~K} / \mathrm{min}$ in a $20-\mathrm{ml} / \mathrm{min}$ flow of dry nitrogen. From the effluent $\mathrm{NH}_{3}$ was absorbed in distilled water. The $\mathrm{pH}$ of the absorbing solution was kept between $\mathrm{pH}=5.5$ and 6.0 by automatically titrating the absorbed $\mathrm{NH}_{3}$ with $0.1 \mathrm{M} \mathrm{HCl}$ solution. The ammonia, evolved from the sample between 423 and $823 \mathrm{~K}$ during TPAE run, was taken as equivalent with the IEC of the sample and the Brønsted acid site concentration of the deammoniated sample.

IR spectra were recorded by a Nicolet FT-IR spectrometer Impact 400 using the wafer transmission technique. The wafers of the catalysts were activated in high vacuum at $673 \mathrm{~K}$ for 1 hour then contacted with pyridine (Py) at $473 \mathrm{~K}$ and 5.7 mbar Py pressure for $30 \mathrm{~min}$, cooled down to $373 \mathrm{~K}$ in Py atmosphere and degassed then at increasing temperatures. The spectra were obtained at room temperature by collecting 32 scans at a resolution of $2 \mathrm{~cm}^{-1}$. Spectra were normalized to wafer thickness of $5 \mathrm{mg} / \mathrm{cm}^{2}$.

\subsection{Catalysis}

For the catalytic test a mixture of ethylbenzene and m-xylene (Merck, 22\%:78\% respectively, variation no more than $1 \%$ ) was prepared. The reaction is carried out in a fixedbed flow-through reactor at atmospheric pressure at $623 \mathrm{~K}$ and $683 \mathrm{~K}$ at various space times. Before the catalytic runs, the catalysts were activated in situ by $\mathrm{N}_{2}$ stream at $823 \mathrm{~K}$ for 2 hours in order to dehydrate the zeolite and to decompose the $\mathrm{NH}_{4}$-form to $\mathrm{H}$-form. Then nitrogen 
carrier gas was passed through a saturator containing the mixed reactant feed and adjusted at temperature of $293.5 \mathrm{~K}$ and the content of the feed was checked by the bypass. Since the boiling points of both feed components are very close, the partial pressure of the mixture (about $0.9 \mathrm{kPa}$ ) and the mol ratio of the ingredients were easily controlled and kept close for a long time. Catalytic data at the $5^{\text {th }}$ min of the reaction onset were considered for the comparison of the catalysts in order to minimize the effect of the deactivation differences. Online analysis of the reaction products was carried out using HP-GC with a $25 \mathrm{~m}$ FFAP capillary column.

\section{Results and discussion}

\subsection{Structural characteristics}

XRD patterns (not shown) indicate that all zeolite samples (ZSM-5, BEA, MCM-22) showed high crystallinity and phase purity except the hierarchical ZSM-5(H) which possess lower $(70 \%)$ crystallinity than that of the microporous ZSM-5(R) [27].

SEM and TEM images of the studied catalysts are presented in Fig. 1. Regular crystallites with disk-like morphology and an average diameter of about $2 \mu \mathrm{m}$ and 0.5-1.0 $\mu \mathrm{m}$ thickness are well distinguished in the SEM photograph of ZSM-5(R), (Fig. 1b), whereas ZSM-5(H) exhibit a sponge-like morphology (Fig. 1a). TEM images shows that BEA(R) consists of square-shape agglomerates with a size of about 200-300 nm (Fig. 1d). The size of the crystals of the BEA(nano) sample is about one magnitude lower than BEA(R), i.e. 30-40 nm (Fig. 1c). The results from SEM represent that the MCM-22 sample (Fig. 1e) is composed of thin, randomly connected platelets with a size of 1-2 $\mu \mathrm{m}$ and a thickness of about 0.1-0.2 $\mu \mathrm{m}$. 

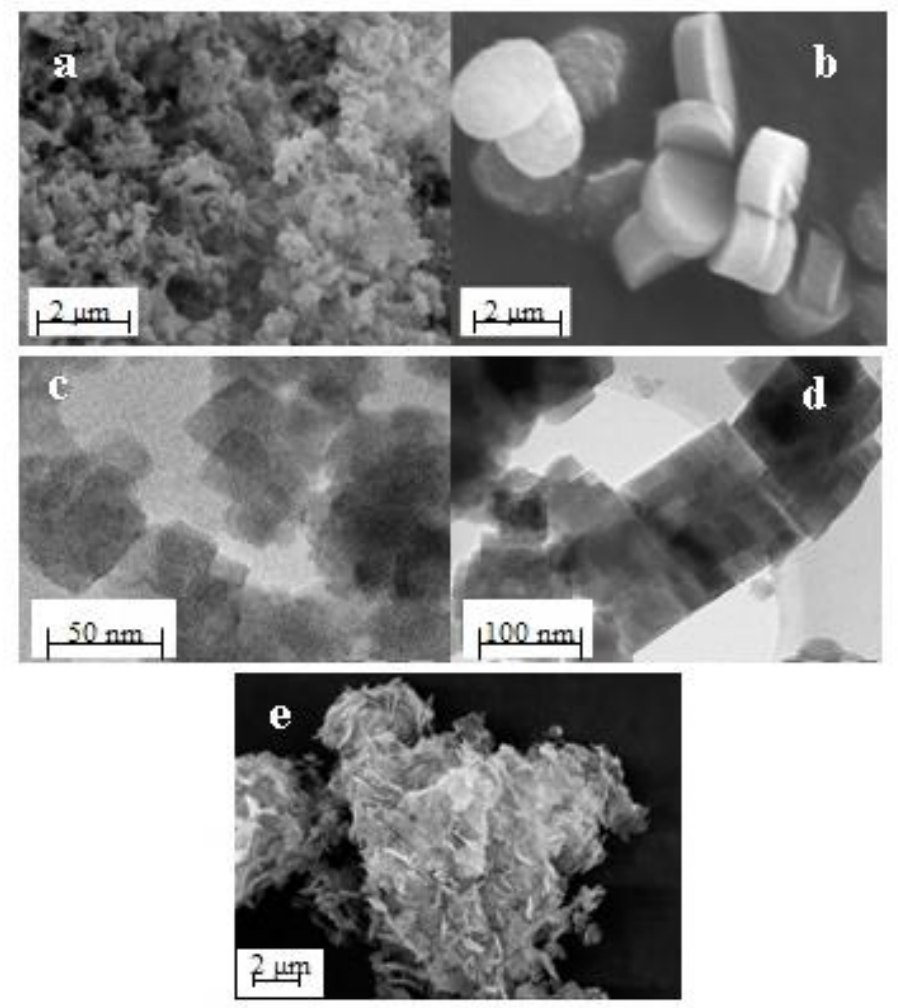

Figure 1. SEM images of (a) ZSM-5(H), (b) ZSM-5(R), TEM images of (c) BEA(nano), (d) BEA(R) and (e) SEM image of MCM-22.

The $\mathrm{N}_{2}$ isotherms suggest that all zeolite materials (that of Beta and MCM-22 not shown) contain micropores that have been saturated with adsorbate at low relative pressure. The increased surface area (Table 1) and the pronounced hysteresis loop on the isotherm of the ZSM-5(H) sample (Figs. 2a, b and c) confirm the pore hierarchy in this modification. The $\alpha_{s}$ plots calculations show (Table 1) that the micropore volume of ZSM-5 (H) is about twice lower than that of ZSM-5 (R). As it was proposed in our previous paper [27], this could be due to the lower crystallinity of the former sample or just on the expense of the presence of mesopores. 


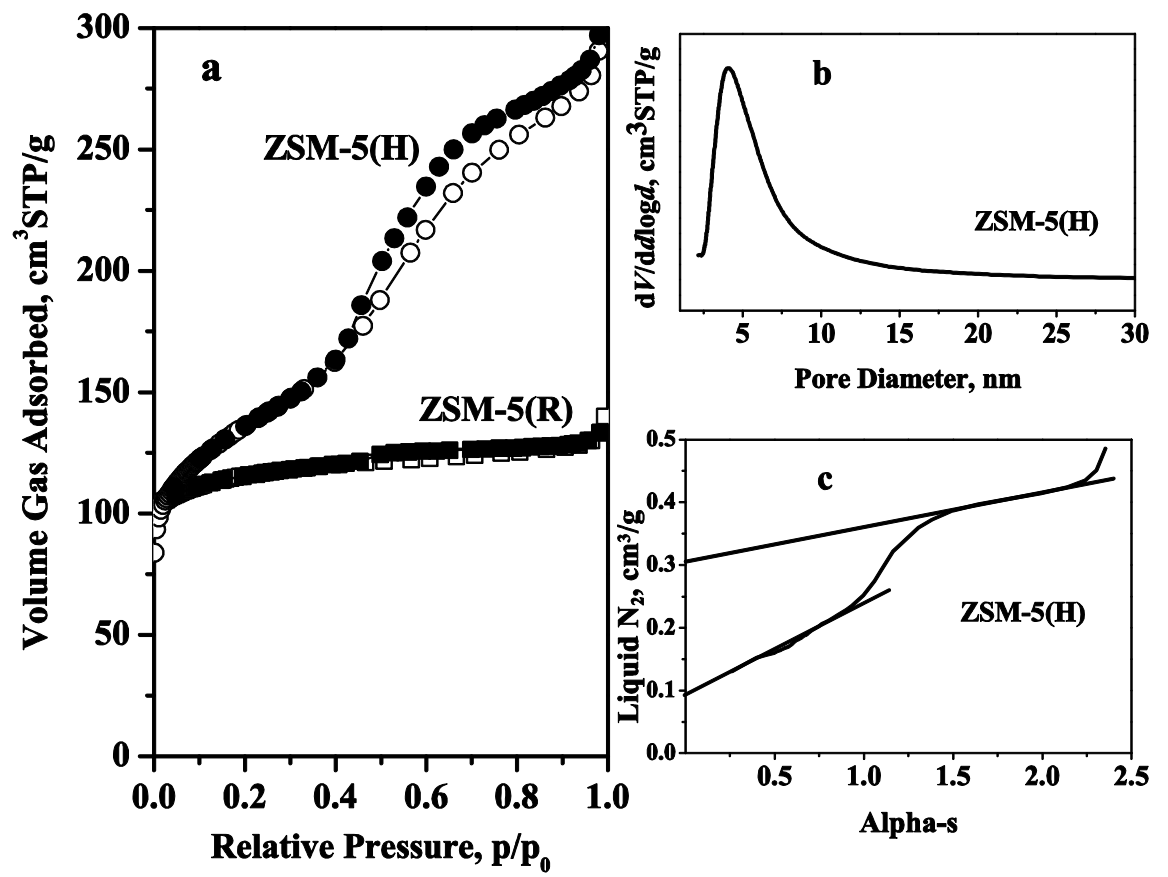

Figure 2. $\mathrm{N}_{2}$ adsorption isotherms of ZSM-5(H) and ZSM-5(R) (a), the pore size distribution of ZSM-5(H) calculated by the BJH method (b) and Alpha-s plot (c).

The hysteresis loop at relative pressure of about 0.9 on the isotherm of the BEA(nano) (not shown) sample suggests that nanocrystalline BEA particles possess small intercrystalline mesoporosity (Table 1).

\subsection{Acidity}

The effect of mesopore formation in zeolite ZSM-5 can be recognized in the FT-IR spectrum of ZSM-5(H) as the band at $3744 \mathrm{~cm}^{-1}$ in the $v_{\mathrm{OH}}$ region, typical for the isolated $\mathrm{SiOH}$ species on the external surface has been increased substantially (Fig. 3a), compared to the conventional reference sample. The substantial increase in the silanol population in the hierarchical sample is due to the formation of intracrystalline mesopores and consequently to the significantly higher specific surface area. The broad band in the region of $3740-3500 \mathrm{~cm}^{-1}$ is ascribed to hydrogen bonded silanol groups associated with local framework vacancies. This band is more intense in the spectra of the hierarchical sample suggesting that its structure is more defective (Fig. 3a). 


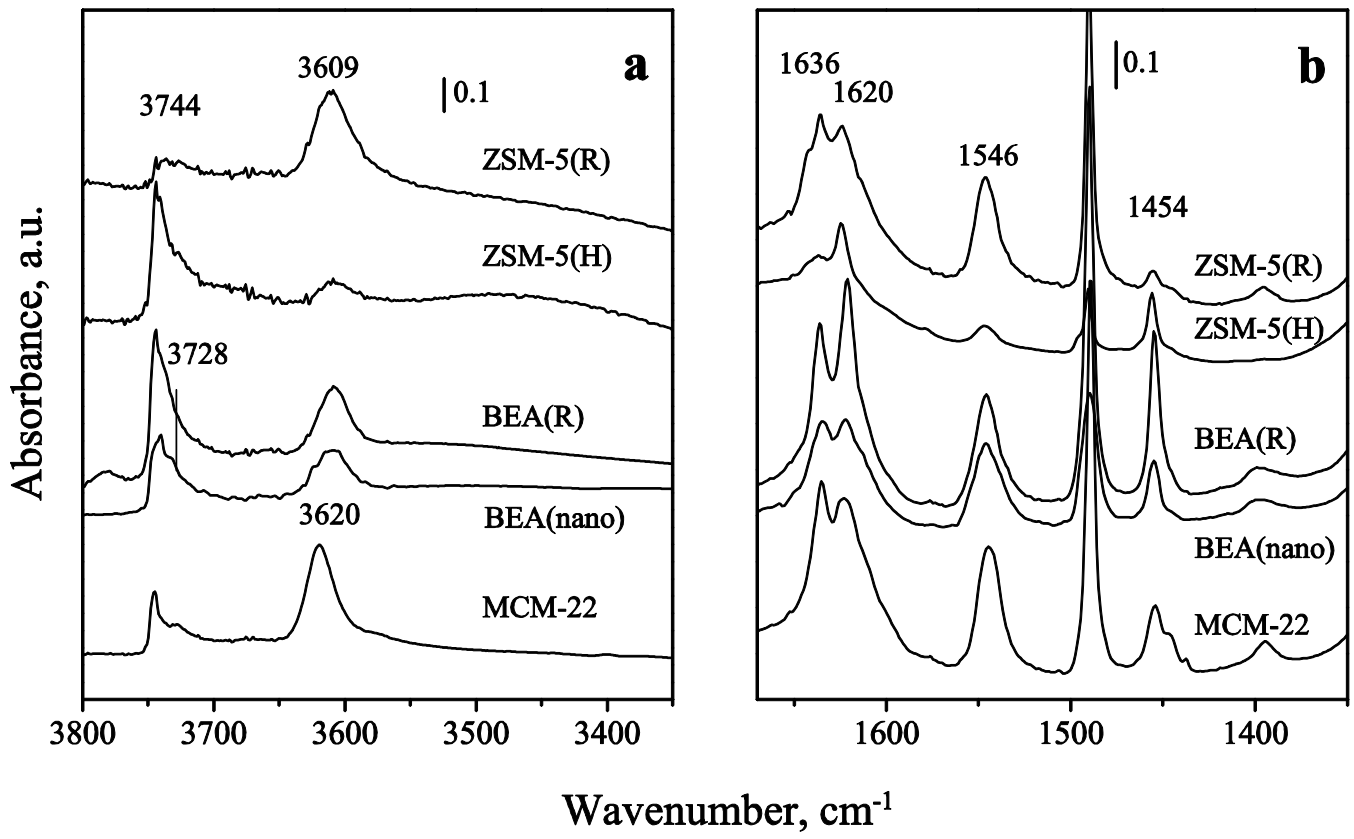

Figure 3. FT-IR spectra of ZSM-5(H), ZSM-5(R), BEA(nano), BEA(R), and MCM-22 in the $v(\mathrm{OH})$ region after evacuation at $673 \mathrm{~K}$ for $1 \mathrm{~h}$ (a) and subsequent Py adsorption at $473 \mathrm{~K}$ and degassing at $473 \mathrm{~K}$ for $0.5 \mathrm{~h}(\mathrm{~b})$.

The IEC of the zeolites, determined as the amount of evolved ammonia, is usually equivalent to the concentration of Broensted acid sites in the generated $\mathrm{H}$-forms. In case of the pentasil samples this statement applies for the common microporous ZSM-5(R) but does not hold for the hierarchical ZSM-5(H) (Table 1, Fig. 3a and b). Obviously, the generation of a mixed micro/mesoporous structure results in loss of crystallinity and, most probably, to extra-framework cationic alumina release, that also reduces the IEC for ammonia of this preparation. The reduction of IEC for ZSM-5(H) is much lower $(\sim 25 \%)$ than the decrease in the intensity of the band at $3610 \mathrm{~cm}^{-1}(\sim 70-80 \%)$ corresponding to its acidic bridging $\mathrm{OH}$ groups (Fig. 3a). This effect has been interpreted by us [27] considering the contribution of the tricoordinated Al sites to the ammonium IEC of the sample. The bands of adsorbed Py appeared in the infrared spectra at $1454 \mathrm{~cm}^{-1}$ and $1620 \mathrm{~cm}^{-1}$ (Fig. 3b) are indeed characteristic for high Lewis acid sites concentration ensued from tricoordinated framework aluminum atoms or octahedrally coordinated extraframework alumina (EFAl) species. Analogous lower Broensted acid sites concentration in the micro-mesoporous ZSM-5 $(\mathrm{H})$ prepared by the same synthesis procedure has been obtained by Suzuki et al. [28]. To circumvent this effect the authors propose a change in the preparation procedure of such hierarchical zeolites by replacing TEOS for other silica sources.

The close values of IEC (Table 1) and the same intensity of the FT-IR bands at 3609 $\mathrm{cm}^{-1}$ (before Py adsorption) and $1546 \mathrm{~cm}^{-1}$ (after Py adsorption) show that the two BEA 
samples possess similar Broensted acidity. However, the strong band at $1454 \mathrm{~cm}-1$ (Fig. 3b) suggests that $\mathrm{BEA}(\mathrm{R})$ having larger crystals contains higher amount of Lewis acid sites than $\operatorname{BEA}($ nano).

FT-IR spectra show that the number of Broensted acid sites in the MCM-22 sample is close to that of the BEA(R) sample (Figs. 3a and b).

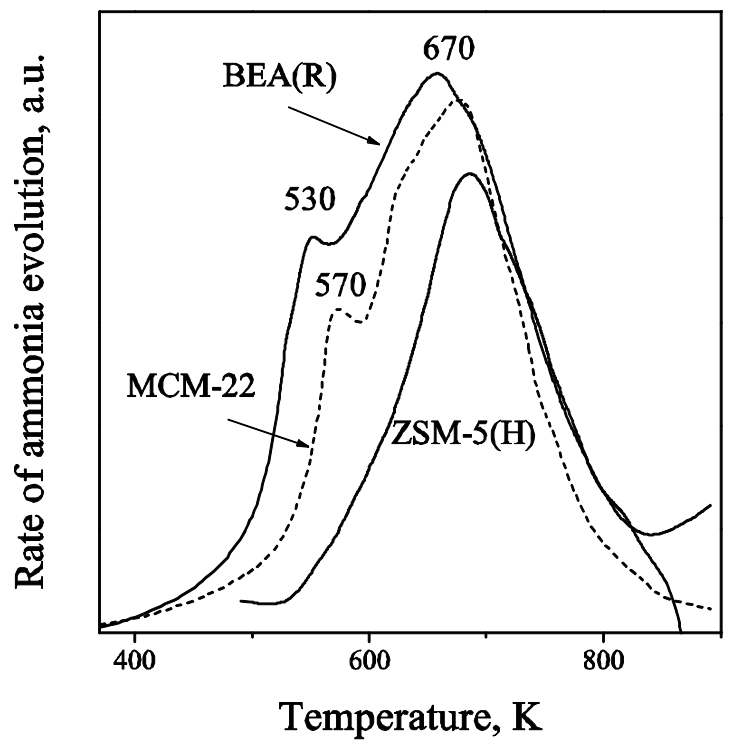

Figure 4. Derivative plots of the temperature-programmed ammonia evolution curves for the $\mathrm{NH}_{4}$-form of ZSM-5(H), BEA(R), and MCM-22 samples.

Derivative plots of the TPDA curve of the catalysts (Fig. 4) have the maximum at about the same temperature $(670 \mathrm{~K})$ suggesting that the three zeolites possess Broensted acid sites with similar strong acidity, but the presence of additional low-temperature peak in the curve of $\mathrm{BEA}(\mathrm{R})(\sim 530 \mathrm{~K})$ and MCM-22 ( $570 \mathrm{~K})$ show that these zeolites contain also Broensted acid sites with weaker acidity.

\subsection{Catalytic performance}

On acid catalysts neat ethylbenzene can undergo two main reactions: disproportionation and dealkylation whereas xylenes transform by isomerisation and disproportionation, the latter reaction being the main source of the undesired xylenes loss $[19,20]$. Transalkylation between xylenes and ethylbenzene can also occur on acid sites in particular from xyleneethylbenzene mixture and also leads to loss of xylenes [20]. Thus, EB conversion and xylene loss together with the percent of p-xylene approach to equilibrium in the final product were 
the main characteristics of the efficiency of the catalysts used for such mixed feed transformation.

Table 2. Products distribution upon ethylbenzene-m-xylene mixed feed conversion over conventional (ZSM-5(R)) and hierarchical ZSM-5 (ZSM-5(H)) $\left(\mathrm{T}_{\text {react. }} 683 \mathrm{~K}\right.$, at the $5^{\text {th }}$ min of TOS).

\begin{tabular}{|c|c|c|c|}
\hline Catalysts & ZSM-5(R) & ZSM-5(H) & ZSM-5(H) \\
\hline Contact time, $\mathrm{h}$ & 0.11 & 0.11 & 0.82 \\
\hline \multicolumn{4}{|l|}{ Products (wt.\%) } \\
\hline Ethylene & 1.73 & 0.14 & 0.62 \\
\hline Benzene & 5.09 & 0.46 & 1.93 \\
\hline Toluene & 0.33 & 0.05 & 0.76 \\
\hline Ethylbenzene & 14.8 & 21.3 & 17.88 \\
\hline Xylenes & 78.05 & 78.05 & 78.26 \\
\hline Trimethylbenzenes & 0.05 & - & 0.07 \\
\hline Ethyltoluenes & - & traces & 0.4 \\
\hline Diethylbenzenes & - & - & - \\
\hline Dimethylethylbenzenes & - & - & 0.06 \\
\hline $\mathrm{C}_{9}^{+}$ & - & - & - \\
\hline EB conversion, $\%$ & 34.1 & 4.7 & 15.2 \\
\hline Xylene loss, \% & - & - & 0.63 \\
\hline $\begin{array}{l}\% \text { p-xylene approach to } \\
\text { equilibrium a }\end{array}$ & 71.0 & 24.6 & 75.7 \\
\hline
\end{tabular}

In our previous study on toluene disproportionation and 1,2,4-trimethylbenzene (TMB) transformation over the same ZSM-5(H) and $-(\mathrm{R})$ couple of samples [27] it was found that with both reactants, the activity of the ZSM-5 zeolite containing mesopores is much lower compared to its conventional counterpart. Analogously, much lower xylene isomerization (pxylene approach to equilibrium of $24.6 \%$ for the hierarchical sample compared to $71 \%$ for ZSM-5(R)) and reduced ethylbenzene dealkylation (4.7\% against $34.1 \%$ respectively) are detected in the present experiments (Table 2). In accordance with the presence of medium pores in these catalysts, together with the strongly decreased number of active Broensted sites, (Fig. 3) they provide very low ability to transfer an alkyl group to the benzene ring as well as to implement the high energy requiring disproportionation reaction. These restrictions reduce strongly the xylene loss on both preparations but, in the same time, inhibit the desired process 
of m-xylene isomerization too. Obviously, the decrease in the concentration of acid sites resulting from the modification procedure of mesopore formation using such type of amphiphilic organosilane as mesopore template appear not effective in preparation of active catalyst for mixed EB-m-xylene feed, irrespective on the low xylene loss detected on them.

Regardless of the catalysts morphology but in correspondence to their close Broensted acidity, both zeolite Beta catalysts reveal analogous catalytic performance at low contact times (Fig. 5 below). The behaviour of this tri-dimensional 12-MR pore zeolite is completely different from

Table 3. Catalytic characteristics of the materials with different structures in the mixed ethylbenzene-m-xylene feed conversion (at the $5^{\text {th }}$ min of TOS).

\begin{tabular}{|c|c|c|c|}
\hline Catalysts & ZSM-5(R) & $\mathrm{BEA}(\mathrm{R})$ & MCM-22 \\
\hline Reaction temperature, $\mathrm{K}$ & 683 & 623 & 683 \\
\hline Contact time, $\mathrm{h}$ & 0.11 & 0.19 & 0.36 \\
\hline Products, wt.\% & & & \\
\hline Ethylene & 1.73 & 1.33 & 2.08 \\
\hline Benzene & 5.09 & 3.63 & 4.31 \\
\hline Toluene & 0.33 & 11.6 & 5.4 \\
\hline Ethylbenzene & 14.8 & 15.29 & 13.66 \\
\hline Xylenes & 78.05 & 59.3 & 74.09 \\
\hline Trimethylbenzenes & 0.05 & 5.98 & 0.16 \\
\hline Ethyltoluenes & - & 2.03 & 0.28 \\
\hline Diethylbenzenes & - & 0.33 & - \\
\hline Dimethylethylbenzenes & - & 0.33 & - \\
\hline $\mathrm{C}_{9}^{+}$ & - & 0.09 & - \\
\hline EB conversion, $\%$ & 34.1 & 37.6 & 38.5 \\
\hline Xylene loss, \% & - & 21.2 & 4.6 \\
\hline $\begin{array}{l}\text { Degree of p-xylene } \\
\text { approach to } \\
\text { equilibrium, } \% \text { a }\end{array}$ & 71.0 & 84.7 & 81.2 \\
\hline
\end{tabular}
isomers) : $25.5 \%$ ( $\mathrm{p}$-xylene equilibrium concentration))x 100 .

that of the ZSM-5 catalysts (Table 3). The comparison of the products distribution shows that the intensity of the side reactions such as methyl and ethyl transfer are extremely high over both BEA samples in accordance with their large pore structure as other authors have observed [21,29]. The vigorous participation of the xylene reactant in these side reactions leads to very high, inadmissible loss of xylene products over both BEA materials. In the interval of contact times up to $0.4 \mathrm{~h}$, almost no effect of the nanosizing on the activity and the 
selectivity can be mentioned (Fig. 5). Considering the toluene to TMB molar ratio (about one, calculated but not presented) it could be concluded that the xylene loss is mainly due to its disproportionation to toluene and TMB at low contact times.

At higher space time, however, substantial increase in the ethylbenzene conversion and, in a certain extent, of the xylene loss is observed (Fig. 5) over the strongly developed nanocrystalline surface of BEA(nano) catalyst. The much higher toluene to TMB ratio (more than two times) is an indication that this growth is mainly connected to the advance of some

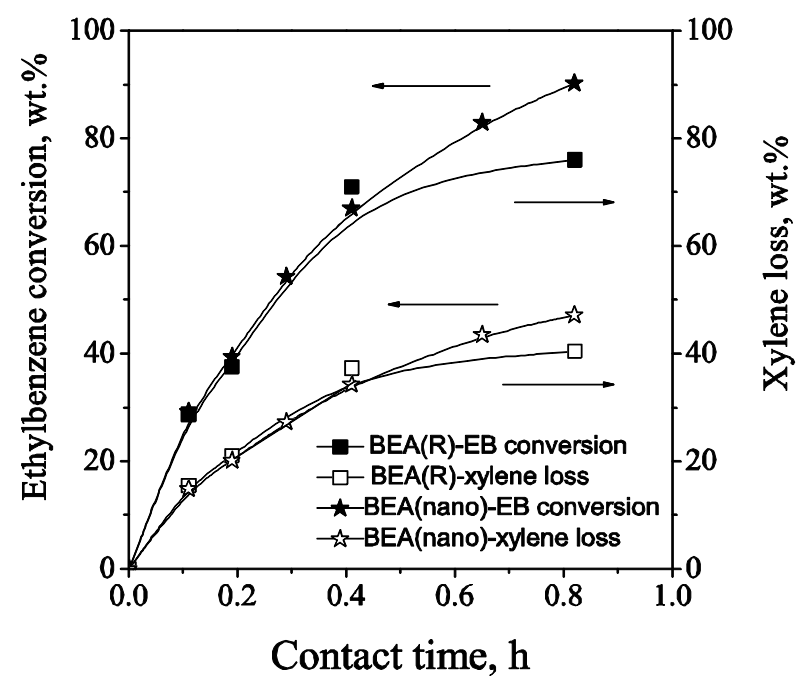

Figure 5. Transformation of mixed $\mathrm{EB}+\mathrm{m}$-xylene feed at the $5^{\text {th }}$ min of the reaction onset in dependence on the contact time over BEA(R) (squares) and BEA(nano) (stars) at $\mathrm{T}_{\text {react. }}=623$

K. (Ethylbenzene conversion - full symbols, xylenes loss - empty symbols).

additional bimolecular reactions engaging EB and xylenes in extra toluene production. These processes need larger space for formation of their bulky intermediates and are facilitated by the enhanced contact time. Transmethylation of ethylbenzene with xylene to ethyltoluene and toluene and a shift of a methyl group from xylene to benzene with formation of two toluene molecules are obviously the reason for this effect as it is suggested in Ref. [19] and [20] respectively. These processes are favoured by the higher residence time and the highly developed surface of the nanocrystallites that ensured enhanced approach to the active sites as Fig. 5 reveals.

To conclude, nanosizing of BEA sample results in increase in the ethylbenzene conversion but only at the highest residence time examined and no beneficial effect on the selectivity towards the xylene products can be found. This result prompts to a compromise that has to be made between the improvement in the EB conversion but, at the same time, to the enhancement of the xylene loss when nano-BEA materials are considered. 
Considering the special features of the catalytic behavior of both catalysts examined above, it could be presumed that zeolite MCM-22 possessing both medium and large pores would reveal predictable and promising properties in the transformation of ethylbenzene-mxylene mixture. Table 3 illustrates the great influence of the zeolite structure on the catalytic action of the studied catalysts. Compared to zeolite BEA, the materials constituted from medium pores need higher reaction temperature and higher contact time to approach close EB conversion rate. The xylene loss over these catalysts, however, is strongly reduced because of the restricted space for the bimolecular reactions generating bulky intermediate products to proceed intensively. The presence of $12 \mathrm{MR}$ surface cups in addition to the 10MR sinusoidal channels in MCM-22 is displayed by the higher yield of side products compared to ZSM-5 (Table 3).

Examinations on the degree of EB conversion and the xylene loss over zeolite MCM-22 for different contact times and two reaction temperatures are presented in Fig. 6. By varying

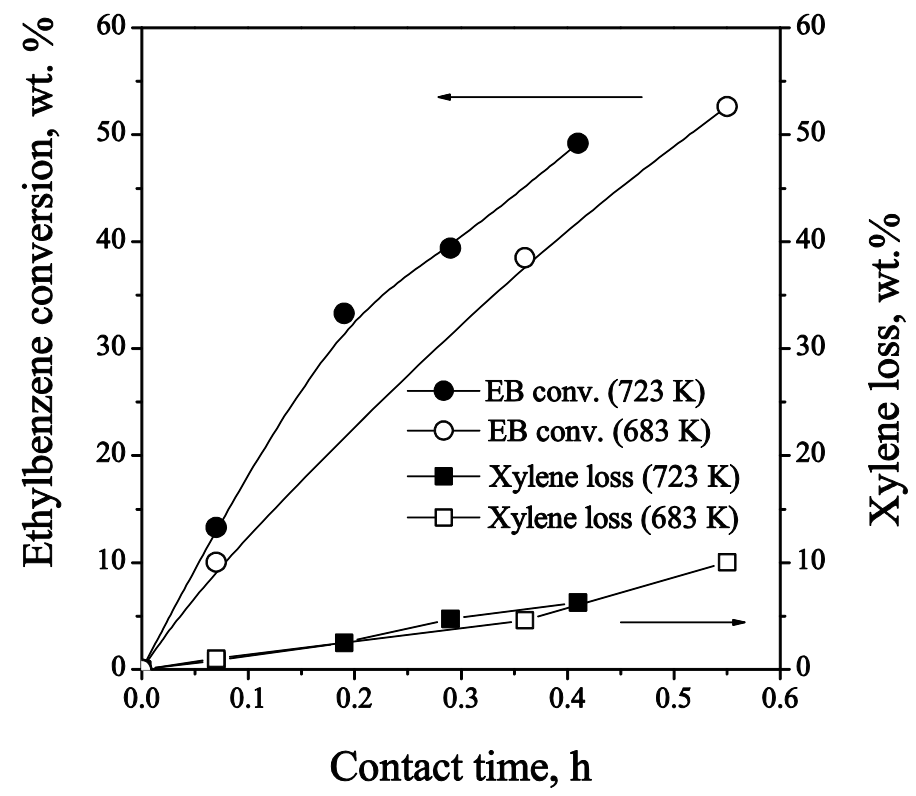

Figure 6. EB conversion and xylene loss for EB-m-xylene reactant mixture transformation at the $5^{\text {th }}$ min TOS over MCM-22 in dependence on the contact time $\left(\mathrm{T}_{\text {react }}=683\right.$ and $\left.723 \mathrm{~K}\right)$.

the reaction temperature and the contact time both EB conversion and xylene loss could be regulated so as to keep the xylene consumption below $5 \%$ that is satisfying for a catalyst with moderate catalytic action between that of the medium and large pore zeolites. Future investigations on the ability to improve the effectiveness of this catalytic reaction by modification of acidity characteristics of MCM-22 and by introduction of metal component for bifunctional action are in progress. 


\section{Conclusion}

Results proved that generation of mesopores in the ZSM-5 crystallites by applying amphiphilic organosilane as mesopore template leads to significant decrease in the Broensted acidity and results in substantial activity decrease in the transformation of EB-m-xylene mixture.

Nanosizing of zeolite BEA raises the degree of EB conversion but the a priori disadvantageously high xylene loss, typical for this material, also increases what makes it inappropriate as catalyst for the conversion of EB-m-xylene mixture.

Each studied zeolite manifests advantages and disadvantages in the catalytic performance, depending primarily on their structure specificity. MCM-22 with its intermediate pore structure between that of ZSM-5 and BEA reveals the most promising properties. At reaction temperature identical to that of the conventional ZSM-5 it displays higher EB conversion and p-xylene approach to equilibrium. Improvement in the catalytic behavior of this material in the transformation of mixed ethylbenzene-m-xylene feed could be attained by development of different approaches to modify its acidity, which is an object of our further investigations.

\section{Acknowledgements}

Support of this work in the framework of the Hungarian-Bulgarian Inter-Academic Exchange Agreement (Project No. 9) is gratefully acknowledged.

\section{References}

[1] T. Hibino, N. Niwa, Y. Murakami, J. Catal. 28, 551 (1991)

[2] E. Benazzi, S. De Tavernier, P. Beccat, C. Nedez, A. Choplin, J.M. Basset, Chemtech 24, 13 (1994)

[3] J. Peres-Ramirez, C.H. Christensen, K. Egeblad, C.H. Christensen, J.C. Groen, Chem. Soc. Rev. 37, 2530 (2008)

[4] S. van Donk, A. Janssen, J. Bitter, K. de Jong, Catal. Rev. - Sci. Eng. 45, 297 (2003)

[5] M. Holm, E. Taarning, K. Egeblad, C.H. Christensen, Catal. Today 168, 3 (2012)

[6] C.H. Christensen, K. Johannsen, I. Schmidt, C.H. Christensen, J. Am. Chem. Soc. 125, 13370 (2003)

[7] F.-S. Xiao, L. Wang, C. Yin, K. Lin, Y. Di, J. Li, R. Xu, D.S. Su, R. Schlogl, T. Yokoi, T. Tatsumi, Angew. Chem. Int. Ed. 45, 3090 (2006) 
[8] Y. Sun, R. Prins, Appl. Catal. A 336, 11 (2008)

[9] Z. Muzilova, N. Zilkova, S.-K. Park, J. Čejka, Top. Catal. 53, 1457 (2010)

[10] C. Fernandez, I. Stan, J.P. Gilson, K. Tomas, A. Vocente, A. Bonilla, J. Perez-Ramirez, Chem. Eur. J. 16, 6224 (2010)

[11] K. Wang, X. Wang, G. Li, Microporous Mesoporous Mater. 94, 325 (2006)

[12] K. Wang, X. Wang, G. Li, Catal. Commun. 8, 324 (2007)

[13] K. Wang, X. Wang, Microporous Mesoporous Mater. 112, 187 (2008)

[14] J.M. Serra, E. Guillon, A. Corma, J. Catal. 227, 459 (2004)

[15] N. Sharanappa, S. Pai, V. Bokade, J. Mol. Catal. A: Gen. 217, 185 (2004)

[16] I. Schmidt, C.H. Christensen, M. Brorson, E. Derouane, C.H. Christensen, US Patent 7411103B2 (2008)

[17] M.K. Rubin, P. Chu, US Patent 4954325 (1990)

[18] S.-H. Park, H.-K. Rhee, Appl. Catal. A 219, 99 (2001)

[19] E. Benazzi, J.M. Silva, M.F. Ribeiro, F.R. Ribeiro, M. Guisnet, in Zeolites: Refined Tools for Designing Catalytic Sites, ed. By L. Bonneviod, S. Kaliaguine (Elsevier, Amsterdam, 1995), p. 393

[20] J.M. Silva, M.F. Ribeiro, F.Ramou Ribeiro, E. Benazzi, M. Guisnet, Appl. Catal. A 125, 1 (1995)

[21] A. Geetha Bhavani, A. Pandarangan, J. Mol. Catal. A: Chem. 267, 209 (2007)

[22] E. Guillon, S. Lacombe, T. Sozinho, P. Magnoux, S. Gnep, P. Moreau, M. Guisnet, Oil Gas Sci. Technol.- Rev. IFP. 64, 731 (2009)

[23] M. Choi, D. Cha, R. Srivastava, C. Vencatesan, D.-H. Choi, R. Ryoo, Nat. Mater. 5, 718 (2006)

[24] R.L. Wadlinger, G.T. Kerr, E.J. Rossinski, US Patent, 3308069 (1967)

[25] R. Ravishankar, D. Bhattacharya, N. Jacob, S. Sivasankar, Microporous Mater. 4, 83 (1995)

[26] R.M. Mihályi, A. Szegedi, F. Lónyi, in Silica and Silicates in Modern Catalysis, ed. by I. Halász (Transword Research Network, Kerala, 2010), p. 213

[27] M.R. Mihályi, M. Kollár, I. Kolev, V. Mavrodinova, in Current Issues in Organic Chemistry, ed. By K. Hadjiivanov, V. Valtchev, S. Mintova, G. Vayssilov (Heron Press, Sofia 2010), p. 200

[28] K. Suzuki, Y. Aoyagi, N. Katada, M. Choi, R. Ryoo, M. Niwa, Catal. Today 132, 38 (2008) 
[29] L.D. Fernandes, J.L.F. Monteiro, E.F, Sousa-Aguilar, A. Martines, A. Corma, J. Catal. 177, 363 (1998) 